\title{
A Connectionist Approach to Speech Understanding
}

Daniel Nehme Müller, Mozart Lemos de Siqueira and Philippe O. A. Navaux

\begin{abstract}
This paper argues that connectionist systems are a good approach to implement a speech understanding computational model. In this direction, we propose SUM, a Speech Understanding Model, which is a software architecture based on recent neurocognitive researches. The SUM's computational implementation applies wavelets transforms to speech signal processing and connectionist models to syntactic parsing and prosodic-semantic mapping. This approach enables a system of analysis by compensation, when the syntactic analysis does not offer a good reliable level, it is possible to evaluate prosodicsemantic analysis, such as in human speech understanding.
\end{abstract}

\section{INTRODUCTION}

The studies of computational systems, which research oral human-computer communication, are commonly called Spoken Language Understanding (SLU) Systems. This kind of system is derived from two joint technologies: Automatic Speech Recognition (ASR) and Natural Language Processing (NLP). These two technologies are complementary. The natural language can help the speech recognition through information on syntax and semantics. Furthermore, the speech recognition can improve the understanding of the language with contextual information, such as the intonation of the words (prosody) [1].

Current SLU systems lack technology based on biological studies and there is large interference of the designer in the system configuration. Therefore, it is necessary a study of the biological processes involved in the speech understanding, which will lead to a coherent and valid computational representation of such processes. Our work supports the possibility of computational systems integration to represent the speech understanding process, which will improve the SLU systems development.

In fact, recent neurocognitive researches present new approaches to base computational representations. The neurocognitive model developed by Friederici [2] demonstrates a parallel and complementary process that is performed by syntactic and semantics analyses. In this direction, we have proposed a new computational architecture to allow a more robust approach to spoken language processing [3] [4].

This work argues that it is possible to unify several computational systems to represent the speech understanding process. Thus, we propose SUM (figure 1), a Speech Understanding Model based on a neurocognitive model of auditory sentence (section III). Through SUM, we search a computational model that works by compensation: when syntactic analysis fails, semantic analysis can be successful, and vice versa.

The autors are with the Institute of Informatics, The Federal University of Rio Grande do Sul, Brazil (email: danielnm,mozart,navaux @inf.ufrgs.br)

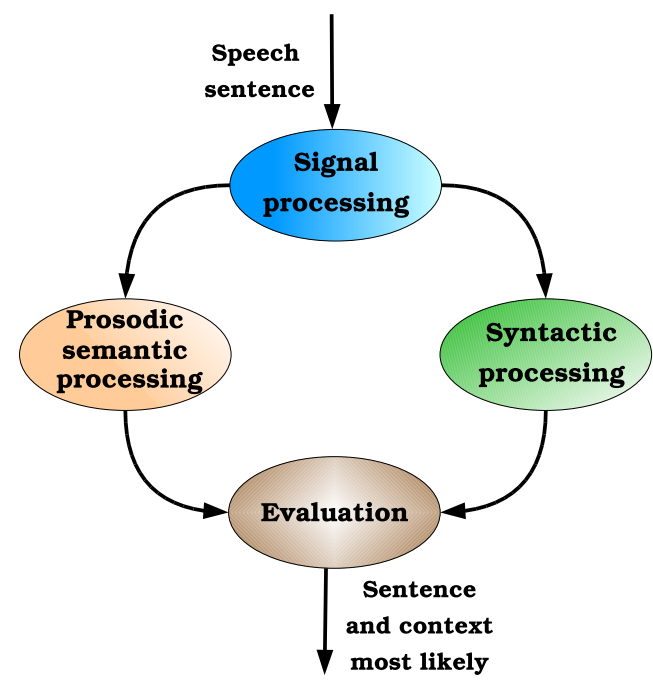

Fig. 1. The Speech Understanding Model - SUM.

In this paper we propose a connectionist implementation of SUM to language processing. It applies wavelet transform to speech signal characteristics extraction. This characteristics are coded to represent the input to connectionist parsing systems. Thus, we present a connectionist solution to syntactic parsing of speech codification in order to define the constructions of the language structures. We also decided to use artificial neural nets to semantic and prosodic analyses, allowing the creation of conceptual maps that will assist on speech contexts definition.

The organization of this paper is as follows: in section II we present the current relevant approaches on SLU systems. The neurocognitive model, basis of SUM, is presented in section III. SUM is described in section IV we here show how wavelet transform is applied to the signal processing and prosodic codification. The connectionist systems used in syntactic parsing and definition of prosodic-semantic contexts are described in section V. In section VI we describe how the operation of the implementation occurs. Finally, in section VII, we discuss the results and new directions of this work.

\section{RELATED WORK}

A SLU system can be seen as the first part of spoken dialog systems, and the second part is composed by sentences generation systems [5]. According to $\mathrm{He}$ and Young [6], a 
SLU system can be divided in three parts: speech recognizer, semantic parser and dialog act decoder.

A widely used method for speech analysis is MFCC (Mel Frequency Cepstral Coefficients), but some limitations have been observed, such as easily noise corruption and a signal frame representation that can hold more than one phoneme [7]. As an alternative, wavelets transforms have been proposed to extract speech signal characteristics by subband division [8][9][10][11], including neural nets [12][13].

Recently, automatic semantic context determination has been the focus of researches, in spite of some work about handmade labeling [14]. This approach is expensive and laborious, however, it permits a full and deep semantic analysis [6][15][16]. On the other hand, the shallow semantic analysis is fully statistical and more robust than deep analysis [6][15][16][17].

Several methods have been developed using shallow semantic analysis approach. All of the works do a classification by distance clustering based on context determination by sentence structure. Self-Organizing Maps (SOMs) were applied to thematic indexing of spoken documents by Kurimo [18]. Pargellis et al. calculated the distance between bigrams to determination of classes [19]. Tur et al. [17] and Haffner [20] implemented algorithms as Boosting and Maximum Entropy to utterances classification. He and Young [6] proposed a HMM (Hidden Markov Model) system with a stack management to encode semantic context, named HVS (Hidden Vector State). Finally, Erdogan [16] and Raymond et al. [11] presented FST (Finite State Transducers) as a solution for generation of semantic hypothesis graphs.

Since the last decade, the prosody has increased its importance to SLU systems. The pioneer work of Kompe [21] presented the importance of accent analysis to word recognition and focus to speech context determination. In this direction, Zhang et al. [22] used the Kompe's concepts to create an automatic focus kernel extraction and to differentiate a pair of words with similar linguistic structure but with unlike meaning. Hasegawa-Johnson et al. [23] demonstrated the relation between accent and sentence structure through a bayesian system of speech recognition. Gallwitz et al. [24] also found in sentences' prosodic limits a new manner to segment and identify words. Lastly, prosody helps in dialog acts determination without independent syntactic and semantic analysis [25] and in games control contextualization [26][27].

\section{Neurocognitive Model}

An adequate evaluation from biologic processes involved in speech understanding will have to lead to a coherent and valid computational representation of these processes. Thus, we found this biologic base at the neurocognitive model of auditory sentence processing proposed by Angela Friederici [2].

Friederici proposes a neurocognitive model of auditory sentence processing that identified which parts of the brain were activated at the time, given the different applied tests. She divided the processing of the auditory sentences in

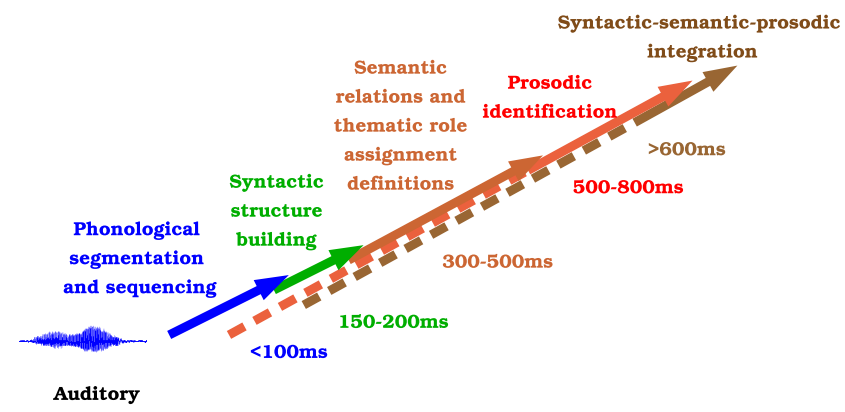

Fig. 2. Improved sequence of neurocognitive model of auditory sentence.

four large phases [2][28]: phonological segmentation and sequencing (up to $100 \mathrm{~ms}$ ); syntactic structure building (between 100 and $300 \mathrm{~ms}$ ); semantic relations and thematic role assignment definitions (between 300 and $500 \mathrm{~ms}$ ); syntacticsemantic-prosodic integration (between 500 and $1000 \mathrm{~ms}$ ). Indeed, the most recent research indicates that the prosody processing description must be added to the neurocognitive model, which occurs at $500 \mathrm{~ms}$ and is combined with other categories at $800 \mathrm{~ms}$ [29]. This process is illustrated in the figure 2 .

In the first phase, an acoustic characteristic extraction and codification is done. In this process, the pitch is isolated and the affective signal is distinguished from the linguistic signal. Thus, the prosodic characteristics, defined by the pitch variation, determine the processing segmentation and form of each part of the signal. The linguistic characteristics will be analyzed at the syntactic level by the left hemisphere of the brain during the second phase [28].

In the second phase, the syntactic analysis is not affected by the prosody and the semantics, and it is processed in an independent manner [30]. The syntactic evaluation process happens where the structure sentence errors cause the need of error corrections without semantic analysis [31]. Moreover, the syntactic processing uses one syntax memory and another for the verbs. This last memory, however, was found only in people who easily reorganized the sentences listened in the tests [32]. Therefore, the syntactic analysis first queries the syntactic memory and also, in some cases, the verbal one.

The semantic analysis is performed in the third phase. In this case, there is a query in the words category memory, which can be observed in tests where the sentences had been organized to produce conflicts related to category and gender [30]. Another study has shown that the semantic processing becomes more important in cases where either syntactic analysis is ambiguous or when hearing is impaired [33]. The semantic analysis apparently awaits the syntactic analysis output in order to solve interpretation problems, brought about mainly by the words' categories contextualization. If the sentences are well structuralized, they will be evaluated by gender, category and semantic context of the involved words [2][28][31].

The fourth and last phase integrates syntax, semantics 
and prosody, necessary to revisit problems not resolved in the previous phases. The syntax structure correction is necessary when lexical terms organization troubles occur [28]. When there is not a consolidated structure to syntactic base, apparently the result of the semantics analysis assists in finding a solution [34].

All these phases presented previously serve as a base for the conception of a computational model. This model tries to understand the four phases verified in Friederici's neurocognitive studies.

\section{SUM: The SPeEch Understanding ModeL}

In order to represent Friederici's neurocognitive model, a computational model is proposed aiming to simulate the speech understanding process [3]. We propose a computational model based on the natural auditory sentence processing to represent the neurocognitive model. From the four described phases in the neurocognitive model, we propose the architecture of SUM, illustrated in the figure 1.

In SUM, the first phase makes the coefficients extraction from speech signal. These coefficients provide the information about the fundamental wave (F0) and they are used in the following phases. The second computational phase is the application of coefficients to proceed the syntactic parsing. In the third phase, the coefficients are used to define semantic contexts. The fourth phase receives the analyses from second and third phases outputs. To each analyzed sentence, the most likely context is indicated.

Besides this architecture, we propose computational methods to its implementation. We apply wavelet transform to characteristics coefficients extraction to speech signal processing. We chose wavelets because they allow a timefrequency speech signal representation.

In the first phase of the computational model, the signal is processed by the application of wavelet transform. The resultant coefficients of this process provide information about the behavior of the F0 and are used as input in the neural nets of the following phases.

The second computational phase is the application of the wavelet coefficients to generation of temporal registers and parsing trees through the system SARDSRN-RAAM previously developed by Mayberry and Miikkulainen [35]. This system is composed by three neural nets: SARDNET - Sequential Activation Retention and Decay Network [36], SRN - Simple Recurrent Network [37] and RAAM - Recursive Auto-Associative Memory [38]. This system generates temporal registers of input words and syntactic trees in the connectionist parsing.

In the third phase, semantic and prosodic maps are applied using the Self-Organizing Map (SOM) [39]. The SOM nets are known for clustering patterns with similar characteristics.

The fourth computational phase performs the reception and analysis of the output of the second and third phases. In this phase, the model indicates the most likely semantic context for a given interpreted sentence.

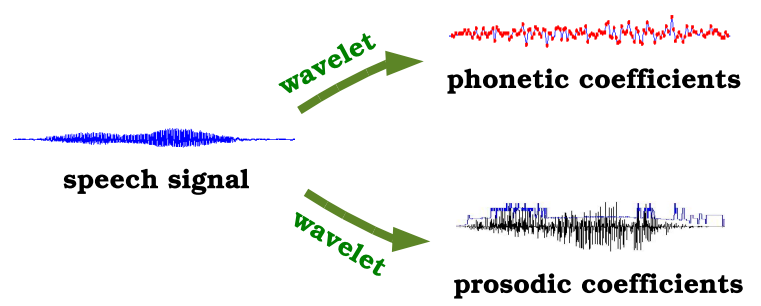

Fig. 3. Coefficients extraction from speech signal.

\section{SUM IMPLEMENTATION}

\section{A. Speech signal processing}

The wavelet transform can be seen as a signal filter, making it possible to build filter banks through them, and, thus, enabling a multiresolution analysis [40]. The wavelet multiresolution analysis is used in this work to extract the characteristics of the speech signal.

The wavelets transforms make wave decompositions in scale (dilation) and temporal displacement (translation). The scale allows the signal differentiation between diverse frequency levels, whereas the translation defines the band wideness in analysis [41]. These parameters permit that high frequencies are analyzed in narrow windows and low frequencies in wide windows.

If we vary only the scale, the wavelets can work as filterbanks with multiresolution analysis [40]. This means that it is possible to obtain more details of signal in different levels of frequency. The scale is defined by

$$
\phi(x)=\sqrt{2} \sum_{k} h_{k} \phi(2 x-k)
$$

where $h_{k}$ is a low-pass filter and $k$ is the scale index. The mother-wavelet will be inserted in this scale by

$$
\psi(x)=\sqrt{2} \sum_{k} g_{k} \psi(2 x-k)
$$

where $g_{k}$ is a high-pass filter.

In this work we use the multiresolution analysis to encode speech signal. This process was split in phonetic and prosodic approaches (fig 3). The phonetic approach is obtained from a single decomposition of wavelet coefficients. The prosodic way is extracted from F0 variation (pitch).

The multiresolution analysis has been used widely as filterbanks to speech signal characteristics extraction by sub-band division [8][12][13][42]. In each sub-band we can extract the spectral density as phoneme identification [9][10][43]. The spectral density $D$ is calculated by

$$
D=\frac{\sum c^{2}}{n}
$$

where $c$ is the sub-band wavelet coefficient and $n$ the number of coefficients. We uses a normalization $D_{N}$ to reduce the variance between coefficients and allows a best performance of neural networks as

$$
D_{N}=\ln (D) .
$$


The composition of all $D_{N}$ calculated from the leaves of the wavelet multiresolution tree are the phonetic coefficients. This coefficients are used as word patterns in connectionist systems to language processing.

According to [44][45], to acquire information on the variations of the F0 speech it is necessary to detect the wavelet maximum points, which correspond to the glottal closure instants (GCI). Several methods have been proposed to improve the maximum point obtained [46][47].

After the maximum points are obtained, we attained the F0 estimation through zero-crossing counting. The prosodic coefficients are the variance of F0 and are used as patterns in prosodic-semantic mapping.

\section{B. Connectionists systems}

The implementation of the processing of the syntactic and prosodic-semantic analysis is done through two systems based on models of artificial neural nets. The deriving signals of linguistic characteristics of wavelets transform are sent to the connectionist system that will perform the syntactic analysis. Those same signals, plus the prosodic coefficients, are sent to the prosodic-semantic analysis system. Our goal is to recognize sentences through both systems simultaneously, as it occurs in Friederici's neurocognitive model, as seen in the section III. Through this recognition, we obtain the most likely phrase, indicated by the most similar context for the sentence presented in the beginning of the processing.

1) Syntactic analysis : The phonetic codification of words, extracted from wavelet transform, is structuralized through the RAAM, whose activation allows the sequencing of the words in the phrase by the SARDSRN-RAAM. Afterward, the temporal sequence of the component words is initiated and the pattern presented in the input layer is distributed to the hidden layer and the SARDNET. This net, in turn, also feeds the hidden layer. Parallel to this hidden layer there is a context layer, characterizing the SRN in the SARDSRN-RAAM. Finally, the output layer generates a pattern sentence that is decoded by the RAAM net. A SARDSRN schema is shown in figure 4.

SARDSRN-RAAM is a shift-reduce parser: RAAM performs a reduce of input and a shift through stacking effect as illustrated in figure 5. SARDSRN makes a temporal register of the input coded by RAAM net, as explained by [35].

The training patterns of this model are sequences of coding words and their desired outputs. The test patterns are sequences of words, not always with meaning and without output information.

A relevant characteristic of the SARDSRN-RAAM is its great capacity to generate parsing sequences. These sequences will allow the training and posterior recognition of multiple parsing trees. These trees are compressed by RAAM net, allowing a robust syntactic analysis.

2) Prosodic-semantic analysis: The concept of semantics applied in this implementation is deriving only of the relations between phonetic and prosodic, without interference of contexts of use of the language. This considered semantic processing is composed by four chained SOM nets based

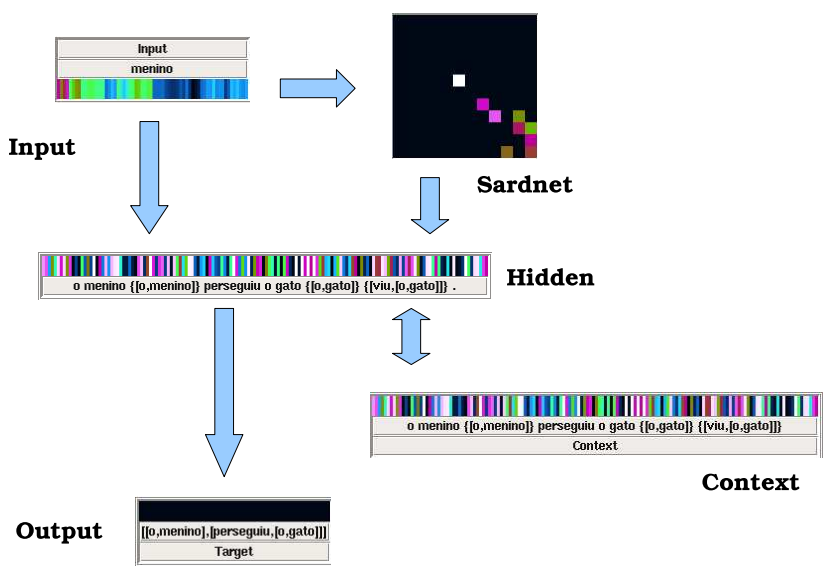

Fig. 4. SARDSRN schema.

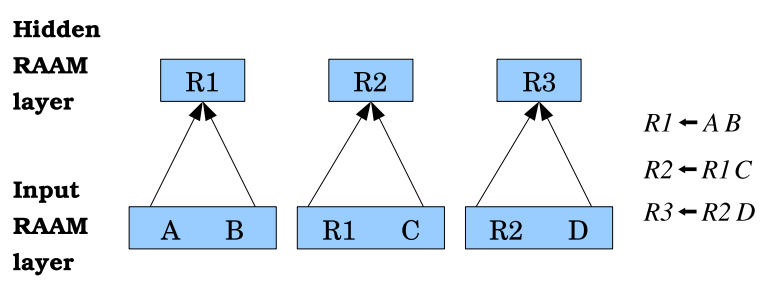

Fig. 5. A example of shift-reduce with RAAM net.

on [48][49][50] (see figure 6). The first SOM net makes a mapping of the prosodic characteristics of the words. The second net performs the learning of the phonetic relations of the words. The third net allows the identification of the semantic words clusters. The fourth net forms sentences clusters based on semantic contexts organized in the third map.

In the first SOM net, a map of prosodic relations of the involved words is formed, whose input is supplied by the prosodic coefficients resulting of wavelet transform. In the second net, another map is obtained with the relations of the phonetic characteristics of each word from the phonetic

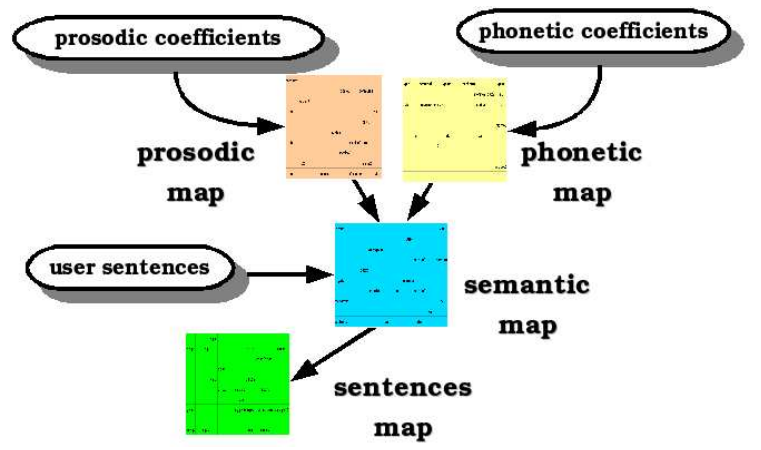

Fig. 6. Maps organization to phonetic, prosodic, semantic and sentences clustering. 


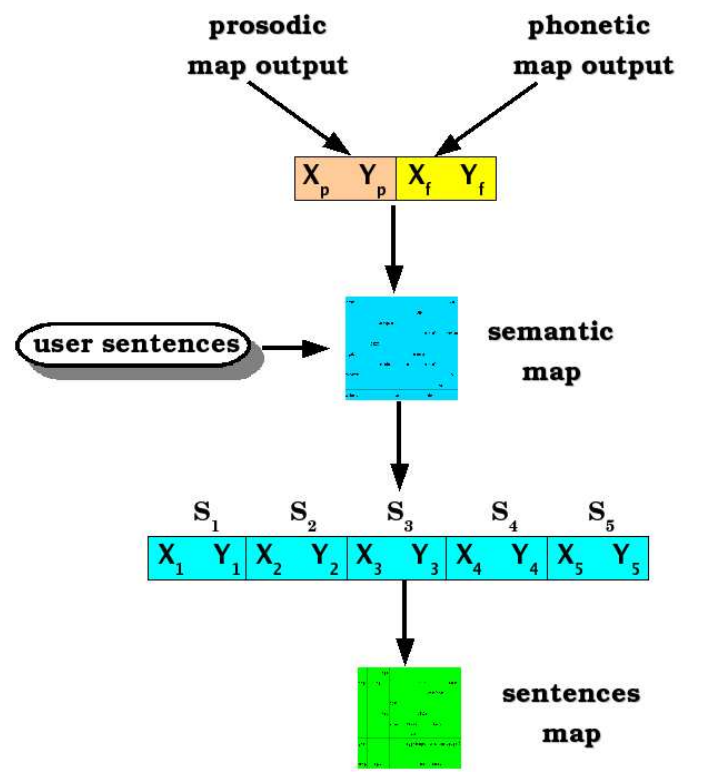

Fig. 7. Input compound in semantic and sentence maps.

coefficients also extracted by wavelet transform. The nets output is sent to the map that does the semantic words clustering. Finally, the last map is responsible for sentence clustering. The sentences are supplied by the user and each word is codified by the preceding maps as input in the sentence map.

The recognition of speech patterns is done in the last map that indicates the similar pattern sentence to the recognized sentence. Indeed, no formalization and definition of linguistic rules is necessary, as it occurs in many conventional natural language systems.

The prosodic map groups the words according to signals derived from the analysis of F0 variations. These analysis give us information on the pauses and accents of the language. The phonetic map of words organizes groups of words according to their linguistic structure and pronunciation. The semantic map uses the indication of the neurons activated in the prosodic map, in addition to the codification of context indicated by the neuron activated in the phonetic map to form word clusters (figure 7). The sentences map makes clustering according to more similar words within a same context. Its input is formed by a compound semantic map response of user sentences as illustrated in figure 7 .

The prosodic and phonetic maps had a high computational cost to train. In this direction, we developed a parallel SOM simulator for maps training. In the parallel SOM algorithm, we divided the map in two branches in each loop that performs neurons access. A typical stretch is as follows:

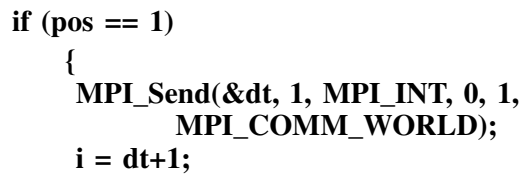

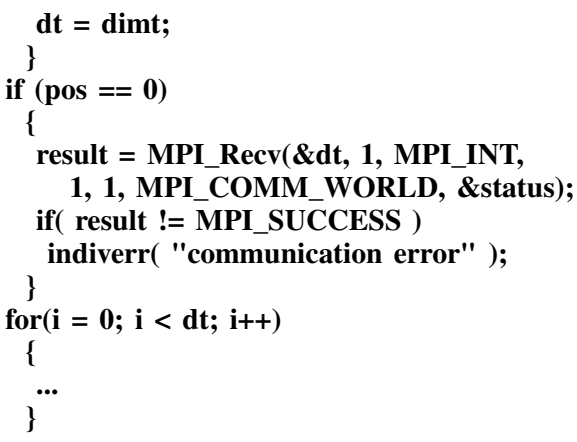

The parallel SOM improved around $40 \%$ the training time. The results are commented in section VI.

\section{Evaluation}

After syntactic and semantic processing, the systems' output is evaluated. The SARDSRN-RAAM system indicates an error rate and the semantic maps system points to the winner neuron in the sentences map. If the syntactic processing has a high error rate, we can do an approximation by semantic processing, and vice versa.

A syntactic error rate lower than 0.5 means a rejection of syntactic structure. A distance in the sentences map higher than 2 means a context rejection. If there is a failure just in the syntactic analysis, the system points out the best sentence of the semantics analysis. If the failure occurs just in the semantics, the system returns the sentence found in the syntactic analysis. If both analyses fail, the sentence is considered incomprehensible. If both have a successful recognition, they are indicated to the user as being good results.

\section{Simulation OF SUM IMPLEMENTATION}

SUM is a software architecture based on Friederici's neurocognitive model, as explained in sections III and IV. Our objective in this work is demonstrate the analysis by compensation of SUM, through its connectionist implementation proposed in section $\mathrm{V}$.

\section{A. Speech signal codification}

We used the Matlab to apply the wavelet transform on the speech signal. We recorded 13 words segmented by hand from 8 sentences spoken by a single Brazilian Portuguese spelling speaker with $22 \mathrm{KHz}$ sampling.

The wavelet transform used was Daubechies' wavelet transforms with eight filter coefficients (daub4 filter in Matlab), and three decomposition levels. We tested all wavelets transforms available to all possible coefficient variations. The election's criterion of the filter was the best signal representation with the lesser number of resultant coefficients. We define a good coefficient wavelet signal representation as the perfect signal reconstruction after inverse transform. The selected level of decomposition (3) corresponds to the band up to $2,7 \mathrm{KHz}$, which allows the representation of the F0 and main formants.

The lexicon was filled with the wavelet coefficients. In this codification, each word is represented by 64 values. 


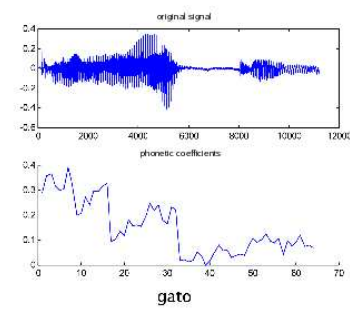

a)

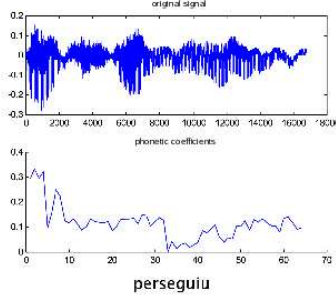

b)
Fig. 8. Generation of phonetic coefficients to word gato (a) and perseguiu (b).

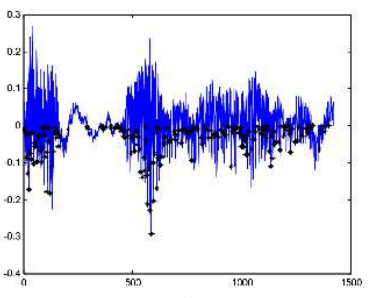

a)

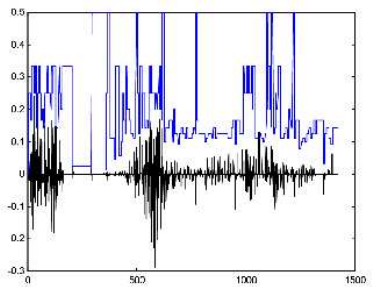

c)

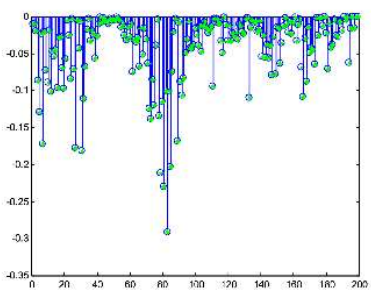

b)

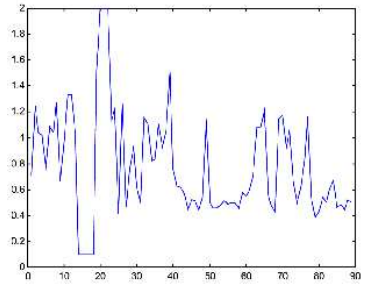

d)
Fig. 9. Generation of prosodic coefficients to word perseguiu: a) maxima points identification; b) cycles between the points calculus; c) cycles $\mathrm{x}$ frequency variation (superior wave); d) obtained coefficients.

These values were filled by hand, as registered by Mayberry III [35]. This is a normal procedure in natural language processing systems, but here we propose the generation of input directly from speech.

As an example, the figure 8 presents the generation of phonetic coefficients and the figure 9 shows the process to obtain the prosodic coefficients. The phonetic coefficients are generated by a direct wavelet decomposition and spectral density calculation. The prosodic coefficients generation have more steps; besides wavelet decomposition, and it is necessary to find maximum points (fig. 9a), identify cycles (fig. $9 b$ ) and frequency from these points (fig. 9c). The obtained frequencies are the prosodic coefficients (fig. 9d).

\section{B. Connectionist systems}

The phonetic coefficients with correspondent words are the lexicon used to SARDSRN-RAAM training. The grammatical relations are represented by written terms and sentences. These input patterns (coefficients and relations) allow the construction of a parsing tree from the RAAM net. In
TABLE I

TRAINED SENTENCES

\begin{tabular}{|c|c|}
\hline acronym & sentence \\
\hline \hline m.v.g. & o menino viu o gato (the boy saw the cat) \\
\hline g.g.m. & o gato gostou da menina (the cat liked the girl) \\
\hline c.m.m. & o cachorro mordeu o menino (the dog bit the boy) \\
\hline m.g.c. & a menina gostou do cachorro (the girl liked the dog) \\
\hline c.p.g. & o cachorro perseguiu o gato (the dog chased the cat) \\
\hline g.v.c. & o gato viu o cachorro (the cat saw the dog) \\
\hline m.m.m. & o menino mordeu a menina (the boy bit the girl) \\
\hline m.p.m. & a menina perseguiu o menino (the girl chased the dog) \\
\hline
\end{tabular}

this codification, each term is represented by 64 values. During RAAM training, new input is added in lexicon. This new input corresponds to RAAM's reduction process. For example, the term $o$ gato (the cat) corresponds to a new input coded in lexicon created by RAAM training.

All SOM maps were implemented with radius 5 and training rate 0.7 . We trained two parallel SOM 10x10 with 64 net inputs to phonetic and prosodic maps. The semantic map was trained in a SOM 10x10 with four inputs to generate sentences from isolated words. These inputs were generated from the composition of the coordinates resulting from the recognition of each word in the prosodic and semantic maps. The sentences map was also built in a SOM 10x10 to obtain the words clustering in a sentence. Each word in a user-defined sentence was coded by the results of prosodicsemantic maps. The words coded were then compounded as input patterns to sentence map. This composition was limited to five words, hence there are ten inputs in the sentence map.

The trained sentences (based on [35]) are in table I. Each indicated item is shown in the figure 11, which presents the clustering of sentences resulting from map training.

As illustration of the functionality of the system, two sentences, not trained, had been elaborated: m.v.g.2 - $a$ menina viu o gato (the girl saw the cat) and c.g.m. - o cachorro gostou da menina (the dog liked the girl). The words are within the known lexicon and we have been using the audio files already existent. The objective of this test is to demonstrate the probable context and syntax guaranteed by the system. The first sentence (m.v.g.2) to be presented to the syntax system presented the sentence a menina perseguiu $o$ gato (the girl chased the cat) as an answer, thus pointing the error of the recognition (fig. 11c). The neurons activation in phonetic, prosodic and semantic maps are represented in figure 10 , respectively. In the sentences map, the identical positioning to the sentence was obtained m.v.g. (fig. 11d).

In the second sentence (c.g.m), the great distance from trained patterns in the sentence map indicates failure in recognition (fig. 11b). On the other hand, the syntactic system returned the exact sentence, although it had not been trained in it (fig. 11a).

These two examples mean that the first sentence corresponded to sentence that had more phonetic representations in common in the trained construction. Meanwhile, in the second sentence the system did not guarantee the semantic recognition, but would identify it in syntactic system. 


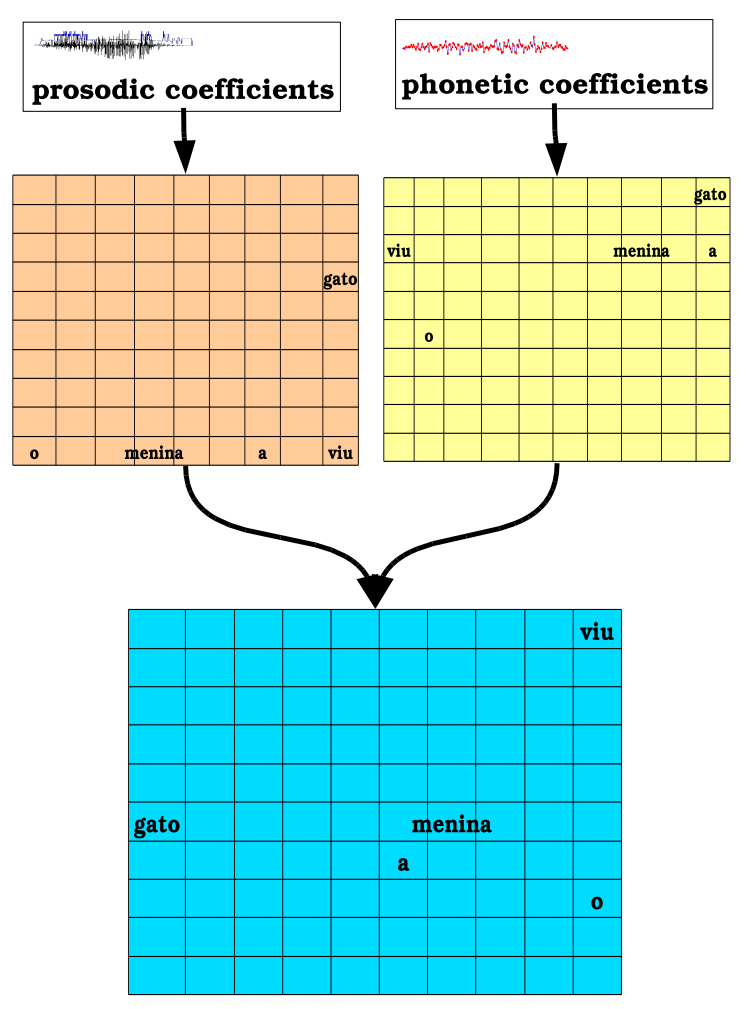

Fig. 10. Sequence of maps to mvg2 (the girl saw the cat) recognition.

a)
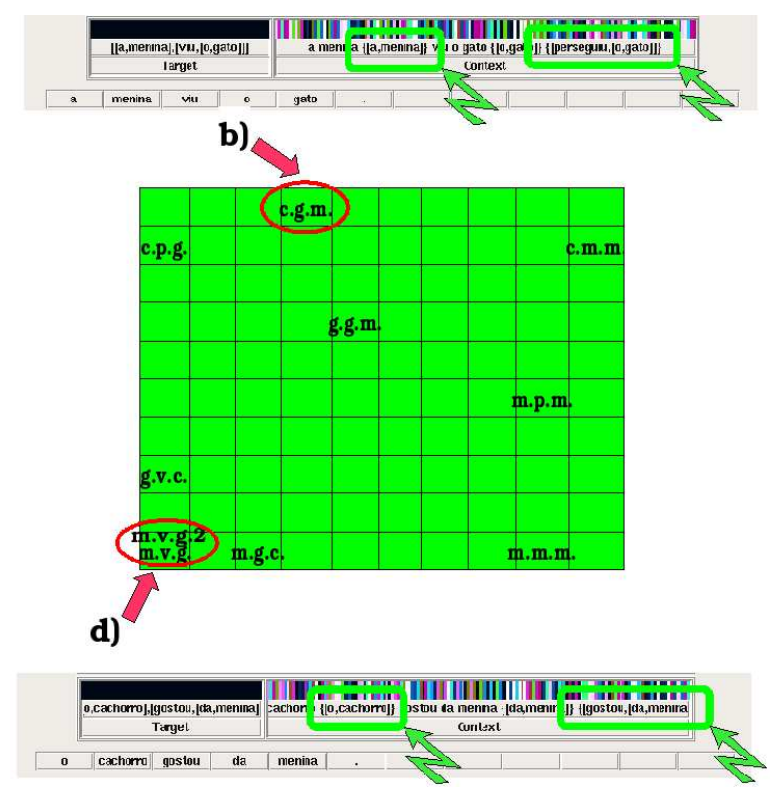

Fig. 11. Sentences recognition in SARDSRN and sentences map: a) and b) c.g.m (the dog liked the girl) sentence and c) and d) m.v.g.2 (the girl saw the cat) sentence.

\section{DISCUSSION AND CONCLUSIONS}

In conclusion, SUM is a computational model to guide the implementation of the auditory sentence process. Our implementation presented a language parsing connectionist approach to SLU systems. The speech signal codification through coefficient wavelets eases the language modeling process. The training of connectionist systems simplifies the work necessary to define grammars and contexts of the language.

The results obtained from wavelet transform allowed a practical codification through the use of the connectionist systems for syntactic and semantics representation. The resultant codification demonstrates that there is an interface between the speech and existent textual parsing connectionists systems.

This opens a new method to implementation of systems for written language. The use of artificial neural nets in the syntactic and prosodic-semantic processing was organized as a facilitator in the language modeling process. The presented neurocognitive model directs to the use of prosodics information as a guide for semantic parsing. It was applied in SUM implementation successfully. In fact, this computational implementation resulted in a system of analysis by compensation, as it occurs in human speech understanding: when the syntactic analysis is not reliable, it is possible to evaluate prosodic-semantic analysis, and vice versa.

The SUM implementation of this work does not intend to be unique, but a beginning for new approaches for real applications. The applied systems have some restrictions to implement robust applications. For example, the SOM maps are static, hindering their expansion to add more words. In such cases, we have been currently researching the Growing SOM (GSOM) [51], Growing Hierarchical SOM (GHSOM) [52] and High Dimensional Growing SOM (HDGSOM) [53]. GSOM was the first method to create a dynamical SOM, but it needs a manual controller for more detailed representations. The newer GHSOM and HDGSOM enable, respectively, a hierarchical and large representations through SOM. Besides this example, we can name the RAAM limitation of data representation, which was improved in Infinite RAAM (IRAAM) [54].

The new SUM implementations will be validated through large corpora and different speakers, as it has been proceeded in similar works. Comparisons between distinct neural networks and linguistic systems must be also evaluated in the future.

\section{REFERENCES}

[1] P. Price, "Spoken language understanding," in Survey of the State of the Art in Human Language Technology, R. A. Cole et al, Ed. Cambridge University Press, Stanford, 1996.

[2] Angela D. Friederici, "Towards a neural basis of auditory sentence processing," Trends in Cognitive Sciences, vol. 6, pp. 78-84, 2002.

[3] Daniel N. Müller and Philippe O. A. Navaux, "Computational model of speech understanding," in Proceedings of the 24th IASTED International Multi-Conference Artificial Intelligence and Applications - AIA'2006, V. Deved, Ed., pp. 97-101. Acta Press, Mar. 2006. 
[4] Daniel N. Müller; Mozart Lemos de Siqueira; Philippe O. A. Navaux, "A model to computational speech understanding," in 7th Workshop on Computational Processing of Written and Spoken Portuguese PROPOR'2006, to be appear.

[5] Ryuichiro Higashinaka et al, "Incorporating discourse features into confidence scoring of intention recognition results in spoken dialogue systems," Speech Communication, vol. 48, pp. 417-436, 2006

[6] Yulan He and Steve Young, "Spoken language understanding using the hidden vector state model," Speech Communication, vol. 48, pp. 262-275, 2006.

[7] Z. Tufekci and J.N. Gowdy, "Feature extraction using discrete wavelet transform for speech recognition," Proc. IEEE Southeastcon 2000, pp. 116-123, 2000.

[8] B. Carnero and A. Drygajlo, "Perceptual speech coding and enhancement using frame-synchronized fast wavelet packet transform algorithms," IEEE Trans. Signal Processing, vol. 47, pp. 1622-1635, June 1999.

[9] K. Kim et al, "Evaluation of wavelet filters for speech recognition," 2000 IEEE Int. Conf. Systems, Man, and Cybernetics, vol. 4, pp. 8-11, 2000.

[10] O. Farooq and S. Datta, "Wavelet based robust sub-band features for phoneme recognition," IEEE Proc. Vision, Image and Signal Processing, vol. 151, pp. 187-193, June 2004.

[11] Christian Raymond et al, "On the use of finite state transducers for semantic interpretation," Speech Communication, vol. 48, pp. 288304, 2006.

[12] M. Gupta and A. Gilbert, "Speech recognition using artificial neural networks," IEEE Workshop Automatic Speech Recognition and Understanding 2001, pp. 445-448, 2001.

[13] E. Avcia et all, "Intelligent target recognition based on wavelet packet neural network," Expert Systems with Applications, vol. 29, pp. 175182, 2005

[14] Ye-Yi Wang and Alex Acero, "Rapid development of spoken language understanding grammars," Speech Communication, vol. 48, pp. 390416, 2006

[15] Nick Webb et al, "Data-driven language understanding for spoken language dialogue," in Proceedings of the AAAI Workshop on Spoken Language Understanding, pp. 23-29. Pittsburgh, 2005.

[16] H. Erdogan et al, "Using semantic analysis to improve speech recognition performance," in Computer Speech and Language, vol. 19, pp. 321-343. Elsevier, 2005

[17] Gokhan Tur; Dilek Hakkani-Tür; Robert E. Schapire, "Combining active and semi-supervised learning for spoken language understanding," Speech Communication, vol. 45, pp. 171-186, 2005.

[18] Mikko Kurimo, "Thematic indexing of spoken documents by using self-organizing maps," Speech Communication, vol. 38, pp. 29-45, 2002.

[19] A. Pargellis et al, "Auto-induced semantic classes," Speech Communication, vol. 43, pp. 183-203, 2004.

[20] Patrick Haffner, "Scaling large margin classifiers for spoken language understanding," Speech Communication, vol. 48, pp. 239-261, 2006

[21] R. Kompe, Prosody in Speech Understanding Systems, SpringerVerlag, Berlin, 1997.

[22] Tong Zhang; Mark Hasegawa-Johnson; Stephen Levinson, "A hybrid model for spontaneous speech understanding," in Proceedings of the AAAI Workshop on Spoken Language Understanding, pp. 60-67. Pittsburgh, 2005.

[23] Mark Hasegawa-Johnson et al, "Simultaneous recognition of words and prosody in the boston university radio speech corpus," Speech Communication, vol. 46, pp. 418-439, 2005.

[24] F. Gallwitz et al, "Integrated recognition of words and prosodic phrase boundaries," Speech Communication, vol. 36, pp. 81-95, 2002.

[25] E. nöth et al, "On the use of prosody in automatic dialogue undestanding," Speech Communication, vol. 36, pp. 45-62, 2002.

[26] H. W. Hastie et al, "Automatically predicting dialogue structure using prosodic features," Speech Communication, vol. 36, pp. 63-79, 2002.

[27] Johann Boye et al, "Robust spoken language understanding in a computer game," Speech Communication, vol. 48, pp. 335-353, 2006.

[28] Angela D. Friederici and Kai Alter, "Lateralization of auditory language functions: A dynamic dual pathway model," Brain and Language, vol. 89, pp. 267-276, 2004.

[29] Korinna Eckstein and Angela D. Friederici, "Late interaction of syntactic and prosodic processes in sentence comprehension as revealed by erps," Cognitive Brain Research, vol. 25, pp. 130-143, 2005.
[30] S. Heim et al, "Distributed cortical networks for syntax processing: Broca's area as the common denominator," Brain and Language, vol. 85, pp. 402-408, 2003.

[31] Sonja Rossi et al, "When word category information encounters morphosyntax: An erp study," Neuroscience Letters, vol. 384, pp. 228-233, 2005.

[32] Sandra H. Vos and Angela D. Friederici, "Intersentential syntactic context effects on comprehension: the role of working memory," Cognitive Brain Research, vol. 16, pp. 111-122, 2003.

[33] Christoph S. Herrmann et al, "The brain generates its own sentence melody: A gestalt phenomenon in speech perception," Brain and Language, vol. 85, pp. 396-401, 2003.

[34] Ina D. Bornkessel et al, "On the cost of syntactic ambiguity in human language comprehension: an individual differences approach," Cognitive Brain Research, vol. 21, pp. 11-21, 2004.

[35] M. R. Mayberry III and Risto Miikkulainen, "SARDSRN: a neural network shift-reduce parser," in Proceedings of IJCAI-99, pp. 820825. Kaufmann, 1999.

[36] Daniel L. James and Risto Miikkulainen, "SARDNET: A selforganizing feature map for sequences," in Advances in Neural Information Processing Systems 7 (NIPS'94, Denver, CO), G. et al Tesauro, Ed., pp. 577-584. MIT Press, 1995.

[37] J.L. Elman, "Finding structure in time," Cognitive Science, vol. 14, pp. 179-211, 1990.

[38] J. B. Pollack, "Recursive distributed representations," Artificial Intelligence, vol. 33, pp. 77-105, 1990.

[39] Teuvo Kohonen, Self-Organization and Associative Memory, SpringerVerlag, 1984

[40] S. G. Mallat, "A theory for multiresolution signal decomposition: The wavelet representation," IEEE Trans. Pat. Anal. Mach. Intell., vol. 11, pp. 674-693, July 1989.

[41] Ingrid Daubechies, Ten lectures on wavelets, Siam, 1992.

[42] Kevin M. Indrebo et al, "Sub-banded reconstructed phase spaces for speech recognition," Speech Communication, inpress.

[43] L.P. Ricotti, "Multitapering and a wavelet variant of mfcc in speech recognition," IEEE Proc. Vision, Image and Signal Processing, vol. 152, pp. 29-35, Feb. 2005

[44] S. Kadambe and G.F. Boudreaux-Bartels, "A comparison of a wavelet transform event detection pitch detector with classical pitch detectors," Twenty-Fourth Asilomar Conference on Signals, Systems and Computers, vol. 2, pp. 1073-1078, 1990.

[45] Shubha. Kadambe and G. Faye Boudreaux-Bartels, "Application of the wavelet transform for pitch detection of speech signals," IEEE Trans. Information Theory, vol. 38, pp. 917-924, 1992.

[46] M. S. Obaidat et al, "Estimation of pitch period of speech signal using a new dyadic wavelet algorithm," Information Sciences, vol. 119, pp. 21-39, 1999.

[47] I. Gavat et al, "Pitch estimation by block and instantaneous methods," International Journal of Speech Technology, vol. 5, pp. 269-279, 2002.

[48] Teuvo Kohonen, "The self-organizing map," Proceedings of the IEEE, vol. 78, no. 9, pp. 1464-1480, 1990.

[49] Risto Miikulainen, Subsymbolic Natural Language Processing, MIT Press, Cambridge, 1993.

[50] Daniel N. Müller and Philippe O. A. Navaux, "Reconhecimento de frases com redes neurais artificiais," in Anais do Terceiro Simpósio Brasileiro de Redes Neurais (III SBRN), Teresa Bernarda Ludermir, Ed., pp. 173-180. UFPE-DI, 1996.

[51] Hans-Ulrich Bauer and Thomas Villmann, "Growing a hypercubical output space in a self-organizing feature map," IEEE Trans. Neural Networks, vol. 8, pp. 218-226, Mar. 1997.

[52] Andreas Rauber et al, "The growing hierarchical self-organizing map: Exploratory analysis of high-dimensional data," IEEE Trans. Neural Networks, vol. 13, pp. 1331-1341, Nov. 2002.

[53] Rasika Amarasiri et al, "Hdgsomr: A high dimensional growing selforganizing map using randomness for efficient web and text mining," in Proceedings of the 2005 IEEE/WIC/ACM International Conference on Web Intelligence (WI'05). 2005.

[54] S. Levy and J. Pollack, "Infinite RAAM: A principled connectionist substrate for cognitive modeling," in ICCM2001. Lawrence Erlbaum Associates, 2001 